\title{
LOCAL ALGEBRAIC APPROXIMATION OF SEMIANALYTIC SETS
}

\author{
M. FERRAROTTI, E. FORTUNA, AND L. WILSON
}

\begin{abstract}
Two subanalytic subsets of $\mathbb{R}^{n}$ are called $s$-equivalent at a common point $P$ if the Hausdorff distance between their intersections with the sphere centered at $P$ of radius $r$ vanishes of order $>s$ when $r$ tends to 0 . In this paper we prove that every $s$-equivalence class of a closed semianalytic set contains a semialgebraic representative of the same dimension. In other words any semianalytic set can be locally approximated of any order $s$ by means of a semialgebraic set and hence, by previous results, also by means of an algebraic one.
\end{abstract}

\section{INTRODUCTION}

In [FFW1] we introduced a notion of local metric proximity between two sets that we called $s$-equivalence: for a real $s \geq 1$, two subanalytic subsets of $\mathbb{R}^{n}$ are $s$-equivalent at a common point $P$ if the Hausdorff distance between their intersections with the sphere centered at $P$ of radius $r$ vanishes of order $>s$ when $r$ tends to 0 .

Given a subanalytic set $A \subset \mathbb{R}^{n}$ and a point $P \in A$, a natural question concerns the existence of an algebraic representative $X$ in the class of $s$-equivalence of $A$ at $P$; in that case we also say that $X$ approximates $A$ of order $s$ at $P$.

The answer to the previous question is in general negative for subanalytic sets which are not semianalytic, even for $s=1$ (see [FFW3]). Furtheremore, in [FFW2 we defined $s$-equivalence of two subanalytic sets along a common submanifold, and studied 1-equivalence of a pair of strata to the normal cone of the pair. By example we showed that a semianalytic normal cone to a linear $X$ may be not 1-equivalent to any semialgebraic set along $X$. It is still an open problem whether a semialgebraic normal cone along a linear $X$ is $s$-equivalent to an algebraic variety along $X$, for all $s$.

On the other hand some partial positive answers were given in [FFW1] and [FFW3]; in particular we proved that a subanalytic set $A \subset \mathbb{R}^{n}$ can be approximated of any order by an algebraic one in each of the following cases:

- $A$ is a closed semialgebraic set of positive codimension,

- $A$ is the zero-set $V(f)$ of a real analytic map $f$ whose regular points are dense in $V(f)$, - $A$ is the image of a real analytic map $f$ having a finite fiber at $P$.

Using the previous results we also obtained that one-dimensional subanalytic sets, analytic surfaces in $\mathbb{R}^{3}$ and real analytic sets having a Puiseux-type parametrization admit an algebraic approximation of any order.

In the present paper we prove that any closed semianalytic set can be locally approximated of any order by a semialgebraic one having the same dimension. Using the main result of [FFW1], it follows that any closed semianalytic set of positive codimension admits an algebraic approximation of any order. Thus we obtain a complete positive answer to our question for the class of semianalytic sets.

Date: July 9, 2012.

2010 Mathematics Subject Classification. Primary 14P15, 32B20, 32S05.

This research was partially supported by M.I.U.R. and by G.N.S.A.G.A. 
The algebraic approximation, elaborating the methods introduced in [FFW3, is obtained by taking sufficiently high order truncations of the analytic functions appearing in a presentation of the semianalytic set.

Finally, let us mention some possible future developments of these notions and ideas. Since we can prove that two subanalytic sets $A, B$ are 1-equivalent if and only if their tangent cones coincide (see also [FFW1]), it would be interesting to extend the notion of tangent cone associating to $A$ a sort of "tangent cone of order $s$ ", say $C_{s}(A)$, in such a way that $A$ and $B$ are $s$-equivalent if and only if $C_{s}(A)=C_{s}(B)$.

There is currently a lot of interest in bilipschitz equivalence of varieties. Most of the work has been in the complex case. Two recent such examples are [BFGR] and [BFGO]. The theory is closely tied up with the notion of the tangent cone, exceptional subcones, and limits of tangent spaces. The real case has been little studied. A good place to start is in the case of surfaces in $\mathbb{R}^{3}$, which is the only real case in which the tangent cone, exceptional lines, and limits of tangent planes have been deeply analyzed (see [OW]). The $s$-equivalence classes are Lipschitz invariants, so they should be a useful tool in this analysis.

\section{BASIC NOTIONS AND PRELIMINARY RESULTS}

If $A$ and $B$ are non-empty compact subsets of $\mathbb{R}^{n}$, we denote by $D(A, B)$ the classical Hausdorff distance, i. e.

$$
D(A, B)=\inf \left\{\epsilon \mid A \subseteq N_{\epsilon}(B), B \subseteq N_{\epsilon}(A)\right\},
$$

where $N_{\epsilon}(A)=\left\{x \in \mathbb{R}^{n} \mid d(x, A)<\epsilon\right\}$ and $d(x, A)=\inf _{y \in A}\|x-y\|$.

If we let $\delta(A, B)=\sup _{x \in B} d(x, A)$, then $D(A, B)=\max \{\delta(A, B), \delta(B, A)\}$.

We will denote by $O$ the origin of $\mathbb{R}^{n}$ for any $n$.

We are going to introduce the notion of $s$-equivalence at a point; without loss of generality we can assume that this point is $O$.

Definition 2.1. Let $A$ and $B$ be closed subanalytic subsets of $\mathbb{R}^{n}$ with $O \in A \cap B$. Let $s$ be a real number $\geq 1$. Denote by $S_{r}$ the sphere of radius $r$ centered at the origin.

(1) We say that $A \leq_{s} B$ if either $O$ is isolated in $A$, or if

(2) $O$ is non-isolated both in $A$ and in $B$ and

$$
\lim _{r \rightarrow 0} \frac{\delta\left(B \cap S_{r}, A \cap S_{r}\right)}{r^{s}}=0 .
$$

(3) We say that $A$ and $B$ are s-equivalent (and we will write $A \sim_{s} B$ ) if $A \leq_{s} B$ and $B \leq_{s} A$.

Observe that if $O$ is non-isolated both in $A$ and in $B$, then

$$
A \sim_{s} B \quad \text { if and only if } \quad \lim _{r \rightarrow 0} \frac{D\left(A \cap S_{r}, B \cap S_{r}\right)}{r^{s}}=0 .
$$

Moreover, if $A \subseteq B$, then $A \leq_{s} B$ for any $s \geq 1$. It is easy to check that $\leq_{s}$ is transitive and that $\sim_{s}$ is an equivalence relationship. The following result shows that $s$-equivalence has a good behavior with respect to the union of sets:

Proposition 2.2. ([FFW3] Let $A, A^{\prime}, B$ and $B^{\prime}$ be closed subanalytic subsets of $\mathbb{R}^{n}$.

(1) If $A \leq_{s} B$ and $A^{\prime} \leq_{s} B^{\prime}$, then $A \cup A^{\prime} \leq_{s} B \cup B^{\prime}$.

(2) If $A \sim_{s} B$ and $A^{\prime} \sim_{s} B^{\prime}$, then $A \cup A^{\prime} \sim_{s} B \cup B^{\prime}$. 
Given a closed subanalytic set $A$ and $s \geq 1$, the problem we are interested in is whether there exists an algebraic subset $Y$ which is $s$-equivalent to $A$; in this case we also say that $Y$ approximates $A$ to order $s$. Evidently the question is trivially true when $O$ is an isolated point in $A$.

Among the partial answers to the previous question that have been already achieved, we recall only the following one which will be used later on:

Theorem 2.3. ([FFW1]) For any real number $s \geq 1$ and for any closed semialgebraic set $A \subset \mathbb{R}^{n}$ of codimension $\geq 1$, there exists an algebraic subset $Y$ of $\mathbb{R}^{n}$ such that $A \sim_{s} Y$.

The following definition introduces a geometric tool which is very useful to test the $s$-equivalence of two subanalytic sets:

Definition 2.4. Let $A$ be a closed subanalytic subset of $\mathbb{R}^{n}, O \in A$; for any real $\sigma>1$, we will refer to the set

$$
\mathcal{H}(A, \sigma)=\left\{x \in \mathbb{R}^{n} \mid d(x, A)<\|x\|^{\sigma}\right\}
$$

as the horn-neighborhood with center $A$ and exponent $\sigma$.

Note that, if $O$ is isolated in $A$, then $\mathcal{H}(A, \sigma)=\emptyset$ near $O$.

Proposition 2.5. ([FFW3] Let $A, B$ be closed subanalytic subsets of $\mathbb{R}^{n}$ with $O \in A \cap B$ and let $s \geq 1$. Then $A \leq_{s} B$ if and only if there exists $\sigma>s$ such that $A \backslash\{O\} \subseteq \mathcal{H}(B, \sigma)$.

The following technical result suggests that horn-neighborhoods can be used to modify a subanalytic set producing subanalytic sets $s$-equivalent to the original one:

Lemma 2.6. Let $X \subset Y \subset \mathbb{R}^{n}$ be closed subanalytic sets such that $O \in X$ and let $s \geq 1$. Then:

(1) for any $\sigma>s$ we have $Y \sim_{s} Y \cup \mathcal{H}(X, \sigma)$;

(2) if $\overline{Y \backslash X}=Y$, there exists $\sigma>s$ such that $Y \backslash \mathcal{H}(X, \sigma) \sim_{s} Y$.

Proof. (a) Since $Y \cup \mathcal{H}(X, \sigma) \subseteq \mathcal{H}(Y, \sigma)$, by Proposition 2.5 for any $\sigma>s$ we have that $Y \cup \mathcal{H}(X, \sigma) \leq_{s} Y$ and hence $Y \cup \mathcal{H}(X, \sigma) \sim_{s} Y$.

(b) Let $\mathcal{U}(X, q)=\left\{x \in \mathbb{R}^{n} \mid \exists y \in X,\|x\|=\|y\|,\|x-y\|<\|x\|^{q}\right\}$.

Arguing as in [FFW1, Corollary 2.6], there exists $q$ such that $Y \backslash \mathcal{U}(X, q) \sim_{s} Y$. Since $X$ and $Y \backslash \mathcal{U}(X, q)$ are subanalytic sets and meet only in $O$, they are regularly situated, i.e. there exists $\beta$ such that $d(x, X)+d(x, Y \backslash \mathcal{U}(X, q))>\|x\|^{\beta}$ for all $x$ near $O$. Then $\mathcal{H}(X, \beta) \subseteq \mathcal{U}(X, q)$ and hence taking $\sigma>\max \{\beta, s\}$ we have that $Y \backslash \mathcal{H}(X, \sigma) \sim_{s} Y$.

Another essential tool will be Łojasiewicz' inequality, which we will use in the following slightly modified version:

Proposition 2.7. Let $A$ be a compact subanalytic subset of $\mathbb{R}^{n}$. Assume $f$ and $g$ are subanalytic functions defined on $A$ such that $f$ is continuous, $V(f) \subseteq V(g), g$ is continuous at the points of $V(g)$ and such that $|g|<1$ on $A$. Then there exists a positive constant $\alpha$ such that $|g|^{\alpha} \leq|f|$ on $A$ and $|g|^{\alpha}<|f|$ on $A \backslash V(f)$.

Proof. The result will be obtained by adapting the proof given by Lojasiewicz under the stronger hypothesis that $g$ is continuous on $A$ (see [Е. Théorème 1]); in that paper he used the following lemma ( $\mathbb{\mathrm { E }}$, Lemma 4]):

if $E \subset[0, \infty) \times \mathbb{R}$ is a compact semianalytic subset of $\mathbb{R}^{2}$ such that $E \cap(\{0\} \times \mathbb{R}) \subseteq$ $\{(0,0)\}$, then there exist positive constants $c, \alpha$ such that $E \subseteq\left\{\left.(x, y) \in \mathbb{R}^{2}|| y\right|^{\alpha} \leq c|x|\right\}$. 
The map $\Phi=(|f|, g): A \rightarrow \mathbb{R}^{2}$ is subanalytic and bounded; hence $\Phi(A)$ is a subanalytic subset of $\mathbb{R}^{2}$ and therefore semianalytic ( $\mathrm{\textrm {E }}$, Proposition 2]). Then $E=\overline{\Phi(A)}$ is a compact semianalytic subset of $[0, \infty) \times \mathbb{R}$.

We have that $E \cap(\{0\} \times \mathbb{R}) \subseteq\{(0,0)\}$ : namely, if $\left(0, y_{0}\right) \in E$, then there exists a sequence $\left\{a_{i}\right\} \subset A$ such that $\lim _{i \rightarrow \infty} \Phi\left(a_{i}\right)=\left(0, y_{0}\right)$ with $a_{i}$ converging to $a_{0} \in A$. By continuity $f\left(a_{0}\right)=0$ and hence $g\left(a_{0}\right)=0$. By the continuity of $g$ at $a_{0}$, we have that $y_{0}=g\left(a_{0}\right)=0$.

So $E$ fulfills the hypotheses of the lemma recalled above and therefore there exist positive constants $c, \alpha$ such that $|g|^{\alpha} \leq c|f|$ on $A$.

Since $|g|<1$, increasing $\alpha$ if necessary we can obtain the thesis.

\section{MAIN THEOREMS}

This section is devoted to the proof of the local approximation theorem for semianalytic sets.

Since $s$-equivalence depends only on the set-germs at $O$, all the sets we will work with will be considered as subsets of a suitable open ball $\Omega$ centered at $O$; we will shrink such a ball whenever necessary without mention.

Definition 3.1. Let $A$ be a closed semianalytic subset of $\Omega$. We will say that $A$ admits a good presentation if the minimal analytic variety $V_{A}$ containing $A$ is irreducible and there exist analytic functions $f_{1}, \ldots, f_{p}$ which generate the ideal $I\left(V_{A}\right)$ and $g_{1}, \ldots, g_{l}$ analytic functions on $\Omega$ such that

$$
A=\left\{x \in \Omega \mid f(x)=O, g_{i}(x) \geq 0, i=1, \ldots, l\right\} .
$$

We start with a preliminary result concerning a way to decompose and present semianalytic sets:

Lemma 3.2. Let $A$ be a closed semianalytic subset of $\Omega$ with $\operatorname{dim}_{O} A=d>0$. Then there exist closed semianalytic sets $\Gamma_{1}, \ldots, \Gamma_{r}, \Gamma^{\prime}$ such that

(1) $A=\left(\bigcup_{i=1}^{r} \Gamma_{i}\right) \cup \Gamma^{\prime}$

(2) for each $i, \operatorname{dim}_{O} \Gamma_{i}=d$ and $\Gamma_{i}$ admits a good presentation

(3) $\operatorname{dim} \Gamma^{\prime}<d$.

Proof. Let $V_{A}$ be the minimal analytic variety containing $A$ (in particular $\operatorname{dim}_{O} V_{A}=d$ ). Let $V_{1} \cup \ldots \cup V_{m}$ be the decomposition of $V_{A}$ into irreducible components. Then $A=$ $W_{1} \cup \ldots \cup W_{m}$ where $W_{i}=A \cap V_{i}$. Then $V_{i}$ is the minimal analytic variety containing $W_{i}$ and $\operatorname{dim}_{O} V_{i}=\operatorname{dim}_{O} W_{i}$.

Each $W_{i}$ is a finite union of sets of the kind $\Gamma=\left\{h_{1}=0, \ldots, h_{q}=0, g_{1} \geq 0, \ldots, g_{l} \geq 0\right\}$.

Let $\Gamma^{\prime}$ be the union, letting $i$ vary, of the $\Gamma$ 's having dimension less than $d$.

For any $\Gamma \subseteq V_{i}$ having dimension $d, V_{i}$ is the minimal analytic variety containing $\Gamma$. It follows that $\Gamma=\left\{f_{1}=0, \ldots, f_{p}=0, g_{1} \geq 0, \ldots, g_{l} \geq 0\right\}$ where $f_{1}, \ldots, f_{p}$ are generators of the ideal $I\left(V_{i}\right)$. Thus we can take as $\Gamma_{1}, \ldots, \Gamma_{r}$ these latter $\Gamma$ 's (letting $i$ vary) suitably indexed.

Notation 3.3. Let $g_{1}, \ldots, g_{l}$ be analytic functions on $\Omega$ and let $f=\left(f_{1}, \ldots, f_{p}\right): \Omega \rightarrow \mathbb{R}^{p}$ be an analytic map. If $A=\left\{x \in \Omega \mid f(x)=O, g_{i}(x) \geq 0, i=1, \ldots, l\right\}$, we will use the following notation:

(1) $A_{i}=\left\{x \in \Omega \mid f(x)=O, g_{i}(x) \geq 0\right\}$ for $i=1, \ldots, l \quad$ (so that $A=\bigcap A_{i}$ )

(2) $b(A)=\bigcup_{i=1}^{l}\left(V\left(g_{i}\right) \cap A\right)$. 
Lemma 3.4. Consider the closed semianalytic set

$$
A=\left\{x \in \Omega \mid f(x)=O, g_{i}(x) \geq 0, i=1, \ldots, l\right\},
$$

where $f: \Omega \rightarrow \mathbb{R}^{p}$ is an analytic map and $g_{1}, \ldots, g_{l}$ are analytic functions on $\Omega$. Assume that $O \in A$. Let $\sigma$ be a real positive number and let $H \subseteq \mathbb{R}^{n}$ be an open subanalytic set such that $H \supseteq \mathcal{H}(b(A), \sigma)$. Then there exists $\eta$ such that, for each $x \in V(f) \backslash(A \cup H)$, there exists $i$ so that $x \notin \mathcal{H}\left(A_{i}, \eta\right)$.

Proof. Since the functions $\sum_{i} d\left(x, A_{i}\right)$ and $d(x, A)$ are subanalytic and vanish exactly on $A$, by Proposition 2.7 there exists $\alpha>0$ such that, for any $x$,

$$
\sum_{i} d\left(x, A_{i}\right) \geq d(x, A)^{\alpha} .
$$

Let $d_{g}$ denote the geodesic distance on $V(f)$.

If $x \in V(f) \backslash A$, we have $d_{g}(x, A)=d_{g}(x, b(A))$. In a suitable closed ball centered at $O$ we can assume that $V(f)$ is connected; hence, by a result of Kurdyka and Orro ([KO]) for any $\epsilon>0$ there exists a subanalytic distance $\Delta(x, y)$ on $V(f)$ such that

$$
\forall x, y \in V(f) \quad 0 \leq \Delta(x, y) \leq d_{g}(x, y) \leq(1+\epsilon) \Delta(x, y) .
$$

Then, if we take for instance $\epsilon=1$,

$$
\forall x \in V(f) \quad 0 \leq \Delta(x, A) \leq d_{g}(x, A) \leq 2 \Delta(x, A)
$$

and so the subanalytic function $\Delta(x, A)$ is continuous at each point of $A$. Hence by Proposition 2.7 there exists $\mu>0$ such that, for any $x$ in $V(f)$,

$$
d(x, A) \geq \Delta(x, A)^{\mu}
$$

and so

$$
\sum_{i} d\left(x, A_{i}\right) \geq \Delta(x, A)^{\mu \alpha} \geq\left(\frac{d_{g}(x, A)}{2}\right)^{\mu \alpha} .
$$

Moreover for any $x \in V(f) \backslash(A \cup H)$ we have that

$$
d_{g}(x, A)=d_{g}(x, b(A)) \geq d(x, b(A)) \geq\|x\|^{\sigma} .
$$

Let us show that the thesis holds choosing $\eta>\sigma \mu \alpha$.

If, for a contradiction, any neighborhood of $O$ contains a point $x \in \bigcap_{i} \mathcal{H}\left(A_{i}, \eta\right) \cap(V(f) \backslash$ $(A \cup H)$, then we have that

$$
\frac{1}{2^{\mu \gamma}}\|x\|^{\sigma \mu \alpha} \leq \sum_{i=1}^{l} d\left(x, A_{i}\right) \leq l\|x\|^{\eta}
$$

which is impossible when $x$ tends to $O$.

For any analytic map $\psi$ defined in a neighborhood of $O$, we will denote by $T^{k} \psi(x)$ the polynomial map whose components are the Taylor polynomials of order $k$ at $O$ of the components of $\psi$.

Lemma 3.5. Let $\varphi$ an analytic function on $\Omega$ such that $\varphi(O)=0$. Let $X$ be a closed semianalytic subset of $\Omega, O \in X$. Then for any real positive $\theta$ there exists $\alpha>0$ such that, for all integers $k>\alpha$, the function $T^{k} \varphi$ has the same sign as $\varphi$ on $X \backslash(\mathcal{H}(X \cap V(\varphi), \theta) \cup\{O\})$. 
Proof. Denote $Z=X \backslash \mathcal{H}(X \cap V(\varphi), \theta)$. Since $V(\varphi) \cap Z=\{O\}$, by Proposition 2.7 there exists $\alpha>0$ such that $\|x\|^{\alpha}<|\varphi(x)|$ for all $x \in Z \backslash\{O\}$.

For all integers $k>\alpha$

$$
\lim _{x \rightarrow O} \frac{\varphi(x)-T^{k} \varphi(x)}{\|x\|^{\alpha}}=0 .
$$

If $O$ is isolated in $Z$, there is nothing to prove. Otherwise assume, for a contradiction, that any neighborhood of $O$ contains a point $x \in Z$ such that $\varphi(x)$ and $T^{k} \varphi(x)$ have different signs (for instance $\varphi(x)>0$ and $T^{k} \varphi(x) \leq 0$ ). Then

$$
\varphi(x)-T^{k} \varphi(x) \geq \varphi(x)>\|x\|^{\alpha}
$$

and hence

$$
\frac{\varphi(x)-T^{k} \varphi(x)}{\|x\|^{\alpha}}>1
$$

arbitrarily near to $O$, which is impossible.

Notation 3.6. Let $_{1}, \ldots, g_{l}$ be analytic functions on $\Omega$ and let $f: \Omega \rightarrow \mathbb{R}^{p}$ be an analytic map. If $A=\left\{x \in \Omega \mid f(x)=O, g_{i}(x) \geq 0, i=1, \ldots, l\right\}$, for any $h, k \in \mathbb{N}$ let

(1) $T^{h}(A)=\left\{x \in \Omega \mid T^{h} f(x)=O, g_{i}(x) \geq 0 i=1, \ldots, l\right\}$

(2) $T_{k}(A)=\left\{x \in \Omega \mid f(x)=O, T^{k} g_{1}(x) \geq 0, \ldots, T^{k} g_{l}(x) \geq 0\right\}$

(3) $T_{k}^{h}(A)=T^{h}\left(T_{k}(A)\right)=\left\{x \in \Omega \mid T^{h} f(x)=O, T^{k} g_{1}(x) \geq 0, \ldots, T^{k} g_{l}(x) \geq 0\right\}$.

Moreover, for any analytic map $\varphi: \Omega \rightarrow \mathbb{R}^{p}$, denote $\Sigma_{r}(\varphi)=\left\{x \in \Omega \mid\right.$ rk $\left.d_{x} \varphi<r\right\}$, and $\Sigma(\varphi)=\Sigma_{p}(\varphi)$

Lemma 3.7. Let $A$ be a closed semianalytic subset of $\Omega$, with $\operatorname{dim}_{O} A=d>0$. Assume that $A=\left\{f(x)=O, g_{i}(x) \geq 0, i=1, \ldots, l\right\}$, with $g_{1}, \ldots, g_{l}$ analytic functions on $\Omega$ and $f: \Omega \rightarrow \mathbb{R}^{n-d}$ an analytic map. Assume also that $\operatorname{dim}_{O}(\Sigma(f) \cap A)<d$ and $\operatorname{dim}_{O} b(A)<d$. Then for any $s \geq 1$ there exist $h_{0}>0, k_{0}>0$ such that, for all integers $h, k$ with $h \geq h_{0}$ and $k \geq k_{0}$, we have
(1) $T_{k}^{h}(A) \leq{ }_{s} A$
(2) $\overline{A \backslash(\Sigma(f) \cup b(A))} \leq_{s} T_{k}^{h}(A)$
(3) $\operatorname{dim}_{O} T_{k}^{h}(A)=d$.

Proof. Let $s \geq 1$ and let $\sigma>s$. Denote $X=(\Sigma(f) \cap A) \cup b(A)$.

(1) Let $H=\mathcal{H}(X, \sigma)$. By Lemma 3.4 there exists $\eta$ such that, for each $x \in V(f) \backslash(A \cup H)$, there exists $i_{0}$ so that $x \notin \mathcal{H}\left(A_{i_{0}}, \eta\right)$.

For all $j$, applying Lemma 3.5 to $V(f), g_{j}$ and $\eta$, we find $\alpha_{1}>0$ such that, for all integers $k>\alpha_{1}$, the functions $g_{j}$ and $T^{k} g_{j}$ have the same sign on $V(f) \backslash\left(\mathcal{H}\left(V(f) \cap V\left(g_{j}\right), \eta\right) \cup\{O\}\right)$.

Let $x \in V(f) \backslash(A \cup H)$. Then $x \notin \mathcal{H}\left(A_{i_{0}}, \eta\right)$ for some $i_{0}$ and hence $g_{i_{0}}(x)<0$; moreover, since $V(f) \cap V\left(g_{i_{0}}\right) \subseteq A_{i_{0}}$, we have that $x \in V(f) \backslash\left(\mathcal{H}\left(V(f) \cap V\left(g_{i_{0}}\right), \eta\right) \cup\{O\}\right)$ and hence, for all integers $k>\alpha_{1}, T^{k} g_{i_{0}}(x)<0$. This implies that $T_{k}(A) \subseteq A \cup H$.

Applying Lemma 2.6 (1) to the sets $X$ and $A$, we have $A \sim_{s} A \cup H$, and so $T_{k}(A) \leq_{s} A$.

Let $B_{k}=\left\{x \in \Omega \mid T^{k} g_{i} \geq 0, i=1, \ldots, l\right\}$.

Since $T_{k}(A)=B_{k} \cap V(f)$, by Proposition 2.7 there exists $\rho>0$ such that $\|f(x)\| \geq$ $d\left(x, T_{k}(A)\right)^{\rho}$ for all $x \in B_{k}$; then for $x \in B_{k} \backslash \mathcal{H}\left(T_{k}(A), \sigma\right)$ we have that $\|f(x)\| \geq\|x\|^{\rho \sigma}$.

Let $h$ be an integer such that $h \geq \rho \sigma$. Then

$$
\lim _{x \rightarrow O} \frac{\left\|f(x)-T^{h} f(x)\right\|}{\|x\|^{\rho \sigma}}=0 .
$$


We have that $T^{h}\left(T_{k}(A)\right) \backslash\{O\} \subseteq \mathcal{H}\left(T_{k}(A), \sigma\right)$; otherwise there would exist a sequence of points $y_{i} \neq O$ converging to $O$ such that $y_{i} \in T^{h}\left(T_{k}(A)\right) \backslash \mathcal{H}\left(T_{k}(A), \sigma\right)$ and hence

$$
\lim _{i \rightarrow \infty} \frac{\left\|f\left(y_{i}\right)-T^{h} f\left(y_{i}\right)\right\|}{\left\|y_{i}\right\|^{\rho \sigma}}=\lim _{i \rightarrow \infty} \frac{\left\|f\left(y_{i}\right)\right\|}{\left\|y_{i}\right\|^{\rho \sigma}} \geq 1
$$

which is a contradiction.

Then by Proposition 2.5 we get that $T_{k}^{h}(A) \leq_{s} T_{k}(A) \leq_{s} A$.

(2) Let $Y=\overline{A \backslash X}$. By our hypotheses $O$ is not isolated in $Y$.

Since $\overline{Y \backslash X}=Y$, applying Lemma 2.6 (2) to the sets $X \cap Y$ and $Y$, up to increasing $\sigma$ we have that $Y \backslash \mathcal{H}(X \cap Y, \sigma) \sim_{s} Y$. Denote

$$
Y^{\prime}=Y \backslash \mathcal{H}(X \cap Y, \sigma) \quad \text { and } \quad H_{i}=\mathcal{H}\left(V\left(g_{i}\right) \cap Y, \sigma\right) .
$$

If for each $i$ we apply Lemma 3.5 to $Y, g_{i}$ and $\sigma$, we can find $\alpha_{2}>0$ such that, for all integers $k>\alpha_{2}$, the functions $g_{i}$ and $T^{k} g_{i}$ have the same sign on $Y \backslash\left(H_{i} \cup\{O\}\right)$.

Since $V\left(g_{i}\right) \cap Y \subseteq X \cap Y$ for each $i$, then $\bigcup H_{i} \subseteq \mathcal{H}(X \cap Y, \sigma)$, and therefore $Y^{\prime} \backslash\{O\} \subseteq$ $\bigcap_{i}\left(Y \backslash\left(H_{i} \cup\{O\}\right)\right)$. In particular

$$
Y^{\prime} \backslash\{O\} \subseteq\left\{T^{k} g_{1}>0, \ldots, T^{k} g_{l}>0\right\} .
$$

From now on, assume that $k>\alpha_{2}$. We will get the result by replacing $f$ with a suitable truncation of it in the presentation of $T_{k}(A)$. We will denote by $B(x, r)$ the open ball centered at $x$ of radius $r$.

By the last inclusion, the distance $d\left(x, b\left(B_{k}\right)\right)$ is subanalytic and positive on $Y^{\prime} \backslash\{O\}$ so, by Proposition 2.7, there exists $\nu>0$ (and we can assume $\nu>s$ ) such that $d\left(x, b\left(B_{k}\right)\right)>$ $\|x\|^{\nu}$ for all $x$ in $Y^{\prime} \backslash\{O\}$. As a consequence

$$
B\left(x,\|x\|^{\nu}\right) \subseteq\left\{T^{k} g_{1}>0, \ldots, T^{k} g_{l}>0\right\} .
$$

Following [FFW3] consider the real-valued function

$$
\Lambda f(x)=\left\{\begin{array}{ll}
0 & \text { if rk } d_{x} f<n-d \\
\inf _{v \perp \operatorname{ker} d_{x} f,\|v\|=1}\left\|d_{x} f(v)\right\| & \text { if rk } d_{x} f=n-d
\end{array} .\right.
$$

Observe that $\Lambda f(x)$ is subanalytic, continuous and positive where $f$ is submersive, in particular on $Y^{\prime} \backslash\{O\}$. Hence, again by Proposition 2.7, there exists $\beta>0$ such that $\Lambda f(x)>\|x\|^{\beta}$ for all $x$ in $Y^{\prime} \backslash\{O\}$.

Consider the subanalytic set $W=\left\{(x, y) \in Y^{\prime} \times \Omega \mid \Lambda f(y) \geq\|x\|^{\beta}\right\}$ and let $W_{0}=$ $\left\{(x, y) \in Y^{\prime} \times \Omega \mid \Lambda f(y)=\|x\|^{\beta}\right\}$; then the set $\left\{(x, x) \mid x \in Y^{\prime} \backslash\{O\}\right\}$ is contained in the open subanalytic set $W \backslash W_{0}$.

The function $\varphi: Y^{\prime} \backslash\{O\} \rightarrow \mathbb{R}$ defined by $\varphi(x)=d\left((x, x), W_{0}\right)$ is subanalytic and positive. Then again by Proposition 2.7 there exists $\tau>0$ (and we can assume $\tau>\nu$ ) such that $\varphi(x)>\|x\|^{\tau}$ on $Y^{\prime} \backslash\{O\}$. Then for all $x \in Y^{\prime} \backslash\{O\}$ and for all $y \in B\left(x,\|x\|^{\tau}\right)$ we have

$$
\|(x, y)-(x, x)\|=\|y-x\|<\|x\|^{\tau}<\varphi(x) .
$$

Hence $(x, y) \in W \backslash W_{0}$, i.e. for all $x$ in $Y^{\prime} \backslash\{O\}$ and for all $y \in B\left(x,\|x\|^{\tau}\right)$ we have $\Lambda f(y)>\|x\|^{\beta}$. In particular $\Lambda f(y)>0$ and hence $d_{y} f$ is surjective for all $y \in B\left(x,\|x\|^{\tau}\right)$.

Let $h$ be an integer such that $h>\beta+1$ and let $\widetilde{f}(x)=T^{h} f(x)$.

Then $T^{h-1} d_{y} f=d_{y} \tilde{f}$; thus we have that $\left\|d_{y} f-d_{y} \widetilde{f}\right\| \leq\|y\|^{h-1}$ for all $y$ near to $O$, where we consider $\operatorname{Hom}\left(\mathbb{R}^{n}, \mathbb{R}^{n-d}\right)$ endowed with the standard norm

$$
\|L\|=\max _{u \neq 0} \frac{\|L(u)\|}{\|u\|} .
$$


Thus by [FFW3, Proposition 3.3] we have

$$
|\Lambda f(y)-\Lambda \widetilde{f}(y)| \leq\|y\|^{h-1}
$$

Claim: for $x \in Y^{\prime} \backslash\{O\}$ and for $y \in B\left(x,\|x\|^{\tau}\right)$, we have

$$
\Lambda \tilde{f}(y) \geq\|x\|^{\beta+1} .
$$

To see this, assume for a contradiction that there exist a sequence $x_{i} \in Y^{\prime} \backslash\{O\}$ converging to $O$ and a sequence $y_{i} \in B\left(x_{i},\left\|x_{i}\right\|^{\tau}\right)$ such that $\Lambda \widetilde{f}\left(y_{i}\right)<\left\|x_{i}\right\|^{\beta+1}$. Thus we have

On the other hand

$$
\frac{\Lambda f\left(y_{i}\right)-\Lambda \widetilde{f}\left(y_{i}\right)}{\left\|x_{i}\right\|^{\beta}}>\frac{\left\|x_{i}\right\|^{\beta}-\left\|x_{i}\right\|^{\beta+1}}{\left\|x_{i}\right\|^{\beta}}=1-\left\|x_{i}\right\| .
$$

$$
\begin{aligned}
& \frac{\Lambda f\left(y_{i}\right)-\Lambda \widetilde{f}\left(y_{i}\right)}{\left\|x_{i}\right\|^{\beta}} \leq \frac{\left\|y_{i}\right\|^{h-1}}{\left\|x_{i}\right\|^{\beta}} \leq \frac{\left(\left\|y_{i}-x_{i}\right\|+\left\|x_{i}\right\|\right)^{h-1}}{\left\|x_{i}\right\|^{\beta}}= \\
& =\left(\frac{\left\|y_{i}-x_{i}\right\|}{\left\|x_{i}\right\|^{q}}+\left\|x_{i}\right\|^{1-q}\right)^{h-1} \leq\left(\left\|x_{i}\right\|^{\tau-q}+\left\|x_{i}\right\|^{1-q}\right)^{h-1}
\end{aligned}
$$

where $q=\frac{\beta}{h-1}$. Since $\tau>1$ and $q<1$, we have that

$$
\frac{\Lambda f\left(y_{i}\right)-\Lambda \widetilde{f}\left(y_{i}\right)}{\left\|x_{i}\right\|^{\beta}}
$$

converges to 0 , which is a contradiction. So the Claim is proved.

Then for all $x \in Y^{\prime} \backslash\{O\}$ the map $\tilde{f}$ is a submersion on $B\left(x,\|x\|^{\tau}\right)$. Hence, using [FFW3, Lemma 3.5], we get $\widetilde{f}\left(B\left(x,\|x\|^{\tau}\right)\right) \supseteq B\left(\widetilde{f}(x),\|x\|^{\lambda}\right)$ with $\lambda=\beta+1+\tau$.

Observe that if $x \in Y^{\prime} \backslash\{O\}$, we have that

$$
\lim _{x \rightarrow O} \frac{\|\tilde{f}(x)\|}{\|x\|^{h}}=\lim _{x \rightarrow O} \frac{\|\tilde{f}(x)-f(x)\|}{\|x\|^{h}}=0 .
$$

So, for any $h \geq \lambda$ and $x \in Y^{\prime}$, the point $O$ belongs to $B\left(\widetilde{f}(x),\|x\|^{\lambda}\right)$ and hence there exists $y \in B\left(x,\|x\|^{\tau}\right)$ such that $\tilde{f}(y)=O$.

Since $\tau>\nu>s$, then $y \in B\left(x,\|x\|^{\nu}\right)$ so that $T^{k} g_{i}(y)>0$ for all $i$, i.e. $y \in T_{k}^{h}(A)$; hence $Y^{\prime} \backslash\{O\} \subseteq \mathcal{H}\left(T_{k}^{h}(A), \lambda\right)$. Then by Proposition 2.5 we have $Y^{\prime} \leq_{s} T_{k}^{h}(A)$ and hence, since $Y^{\prime} \sim_{s} Y$, we have that

$$
\overline{A \backslash(\Sigma(f) \cup b(A))}=Y \leq_{s} T_{k}^{h}(A) .
$$

Therefore, taking $h_{0}=\max \{\rho \sigma, \lambda\}$ and $k_{0}=\max \left\{\alpha_{1}, \alpha_{2}\right\}$, we have the thesis.

(3) The previous argument shows that, for all $h \geq h_{0}$ and $k \geq k_{0}$, there exist points $y \in V\left(T^{h} f\right)$ arbitrarily near to $O$ where $T^{h} f$ is submersive and such that $T^{k} g_{i}(y)>0$ for all $i$. Hence $\operatorname{dim}_{O} T_{k}^{h}(A)=d$.

Theorem 3.8. Let $A$ be a closed semianalytic subset of $\Omega$ with $O \in A$. Then for any $s \geq 1$ there exists a closed semialgebraic set $S \subseteq \Omega$ such that $A \sim_{s} S$ and $\operatorname{dim}_{O} S=\operatorname{dim}_{O} A$.

Proof. We will prove the thesis by induction on $d=\operatorname{dim}_{O} A$.

If $d=0$ the result holds trivially. So let $d \geq 1$ and assume that the result holds for all semianalytic germs of dimension less that $d$.

By Lemma 3.2, by Proposition 2.2 and by the inductive hypothesis, we can assume that

$$
A=\left\{x \in \Omega \mid f(x)=O, g_{i}(x) \geq 0, i=1, \ldots, l\right\}
$$


with $f=\left(f_{1}, \ldots, f_{p}\right)$ such that $V(f)$ is irreducible, $V(f)$ is the minimal analytic variety containing $A$ and $f_{1}, \ldots, f_{p}$ generate the ideal $I(V(f))$. In particular $\operatorname{dim}_{O}\left(\Sigma_{n-d}(f) \cap A\right)<$ $d$; moreover, removing from the previous presentation of $A$ the inequalities $g_{i}(x) \geq 0$ where $g_{i}$ vanishes identically on $A$ (if any), we can assume that $\operatorname{dim}_{O} b(A)<d$.

If $p=n-d$, the thesis follows easily by using Lemma 3.7. In general $p$ can be bigger than $n-d$; in this case we introduce a semianalytic set $\widetilde{A}$ of dimension $d$ which is $s$ equivalent to $A$ and which satisfies the hypotheses of Lemma 3.7. In order to prove the thesis it will be sufficient to approximate $\widetilde{A}$ by means of a semialgebraic set having the same dimension.

Denote by $\Pi$ the set of surjective linear maps from $\mathbb{R}^{p}$ to $\mathbb{R}^{n-d}$ and consider the smooth map $\Phi:\left(\mathbb{R}^{n}-V(f)\right) \times \Pi \rightarrow \mathbb{R}^{n-d}$ defined by $\Phi(x, \pi)=(\pi \circ f)(x)$ for all $x \in \mathbb{R}^{n}-V(f)$ and $\pi \in \Pi$.

The map $\Phi$ is transverse to $\{O\}$ : namely the partial Jacobian matrix of $\Phi$ with respect to the variables in $\Pi$ (considered as an open subset of $\left.\mathbb{R}^{p(n-d)}\right)$ is the $(n-d) \times p(n-d)$ matrix

$$
\left[\begin{array}{ccccc}
f(x) & 0 & 0 & \ldots & 0 \\
0 & f(x) & 0 & \ldots & 0 \\
\vdots & & & & \\
0 & 0 & 0 & \ldots & f(x)
\end{array}\right]
$$

thus, for all $x \in \mathbb{R}^{n}-V(f)$ and for all $\pi \in \Pi$ the Jacobian matrix of $\Phi$ has rank $n-d$.

As a consequence, by a well-known result of singularity theory (see for instance [BK, Lemma 3.2]), we have that the map $\Phi_{\pi}: \mathbb{R}^{n}-V(f) \rightarrow \mathbb{R}^{n-d}$ defined by $\Phi_{\pi}(x)=\Phi(x, \pi)=$ $(\pi \circ f)(x)$ is transverse to $\{O\}$ for all $\pi$ outside a set $\Gamma \subset \Pi$ of measure zero and hence $\pi \circ f$ is a submersion on $V(\pi \circ f) \backslash V(f)$ for all such $\pi$.

Furthermore, let $x \in V(f)$ be a point at which $f$ has rank $n-d$; then there is an open dense set $U \subset \Pi$ such that for all $\pi \in U$ the map $\pi \circ f$ is a submersion at $x$, and hence off some subvariety of $V(f)$ of dimension less than $d$.

Thus, if we choose $\pi_{0} \in(\Pi \backslash \Gamma) \cap U$, the map $F=\pi_{0} \circ f$ has $n-d$ components, $\Sigma(F) \cap V(F) \subseteq V(f) \subseteq V(F), \operatorname{dim}_{O} V(F)=d$ and $\operatorname{dim}_{O}(\Sigma(F) \cap V(F))<d$. In particular $V(f)$ is an irreducible component of $V(F)$.

For each $m \in \mathbb{N}$ denote $\widetilde{A}_{m}=\left\{F=0,\|x\|^{2 m}-\|f\|^{2} \geq 0, g_{i}(x) \geq 0, i=1, \ldots, l\right\}$.

Since $A \subseteq \widetilde{A}_{m} \subseteq V(F)$, we have that $A \leq_{s} \widetilde{A}_{m}$ and $\operatorname{dim}_{O} \widetilde{A}_{m}=d$.

We claim that there exists $m$ such that $\widetilde{A}_{m} \sim_{s} A$; to show that it is sufficient to prove that there exists $m$ such that $\widetilde{A}_{m} \leq_{s} A$. Namely, let $B=\left\{g_{i}(x) \geq 0, i=1, \ldots, l\right\}$. Since $V(\|f\|) \cap B=V(d(x, A)) \cap B$, by Proposition 2.7 there exists $q$ such that $d(x, A)^{q} \leq\|f(x)\|$ for all $x \in B$. Let $m>s q$. Then $d(x, A) \leq\|f(x)\|^{\frac{1}{q}} \leq\|x\|^{\frac{m}{q}}$ for all $x \in \widetilde{A}_{m}$, i.e. $\widetilde{A}_{m} \subseteq \mathcal{H}\left(A, \frac{m}{q}\right)$ and hence $\widetilde{A}_{m} \leq_{s} A$.

Fix $m$ as above and let $\widetilde{A}=\widetilde{A}_{m}$. Let also $\widetilde{X}=(\Sigma(F) \cap \widetilde{A}) \cup b(\widetilde{A})$.

Observe that $b(\widetilde{A}) \cap A=b(A)$ and so $\widetilde{X} \cap A=(\Sigma(F) \cap A) \cup b(A)$.

Denote $K=\widetilde{X} \cap(\widetilde{A} \backslash A)$ so that $\widetilde{X}=(\widetilde{X} \cap A) \cup K$.

By Lemma 3.7 there exist positive integers $h, k$ such that

$$
\overline{\widetilde{A} \backslash \widetilde{X}} \leq_{s} T_{k}^{h}(\widetilde{A}) \leq_{s} \widetilde{A} \quad \text { and } \quad \operatorname{dim}_{O} T_{k}^{h}(\widetilde{A})=d .
$$

Since $\operatorname{dim}_{O}(\widetilde{X} \cap A)<d$, by induction there exists a semialgebraic set $S_{0}$ such that $S_{0} \sim_{s} \tilde{X} \cap A$ and $\operatorname{dim}_{O} S_{0}<d$. Moreover, since $A \subseteq \overline{\widetilde{A} \backslash K} \subseteq \widetilde{A}$, we have that $\overline{\widetilde{A} \backslash K} \sim_{s} \widetilde{A}$. 
Then

$$
\widetilde{A} \sim_{s} \overline{\widetilde{A} \backslash K}=\overline{\widetilde{A} \backslash \widetilde{X}} \cup(\widetilde{X} \cap A) \leq_{s} T_{k}^{h}(\widetilde{A}) \cup S_{0} \leq_{s} \widetilde{A} \cup(\widetilde{X} \cap A)=\widetilde{A}
$$

so we can choose $S=T_{k}^{h}(\widetilde{A}) \cup S_{0}$.

From Theorem 3.8 and from Theorem 2.3 we immediately obtain:

Theorem 3.9. Let $A$ be a closed semianalytic subset of $\Omega$ of codimension $\geq 1$ with $O \in A$. Then for any $s \geq 1$ there exists an algebraic set $Y \subset \mathbb{R}^{n}$ such that $A \sim_{s} Y$.

\section{REFERENCES}

[BFGO] L. Birbrair, A. Fernandes, V. Grandjean, D. O'Shea: Choking horns in Lipschitz Geometry of Complex Algebraic Varieties. arXiv:1206.3105

[BFGR] J.-P. Brasselet, A. Fernandes, N. G. Grulha Jr and M. A. S. Ruas: The Nash modifications and the bi-Lipschitz equivalence. arXiv:1206.5153

[BK] J. W. Bruce and N. P. Kirk: Generic projections of stable mappings. Bull. Lond. Math. Soc., vol. 32 , n. 6 (2000), pp. $718-728$

[FFW1] M. Ferrarotti, E. Fortuna And L. Wilson: Local approximation of semialgebraic sets. Ann. Sc. Norm. Super. Pisa Cl. Sci. (5), vol. I, n. 1 (2002), pp. 1-11

[FFW2] M. Ferrarotti, E. Fortuna And L. Wilson: Approximation of subanalytic sets by normal cones. Bull. Lond. Math. Soc., vol. 39 n. 2 (2007), pp. 247-254

[FFW3] M. Ferrarotti, E. Fortuna and L. WiLson: Algebraic approximation of germs of real analytic sets. Proc. Amer. Math. Soc., vol. 138, n. 5 (2010), pp. 1537-1548

[KO] K. Kurdyka and P. Orro: Distance géodésique sur un sous-analytique. Rev. Mat. Univ. Complut. Madrid, vol. 10 (1997), pp. 173-182

[E] S. LojAsiewicz: Sur la séparation régulière. Geometry seminars 1985, pp. 119-121, Univ. Stud. Bologna, Bologna (1986)

[OW] D. O'Shea and L. Wilson: Limits of Tangent Spaces to Real Surfaces. Amer. J. of Math., vol. 126, n. 5 (2004), pp. $951-980$

Dipartimento di Matematica, Politecnico di Torino, Corso Duca degli Abruzzi 24, I-10129 TORINO, ITALY

E-mail address: ferrarotti@polito.it

Dipartimento di Matematica, Università di Pisa, Largo B. Pontecorvo 5, I-56127 Pisa, ITALY

E-mail address: fortuna@dm.unipi.it

Department of Mathematics, University of Hawail, Manoa, Honolulu, hi 96822, USA

E-mail address: les@math.hawaii.edu 